\title{
State Estimation Over Packet Dropping Networks Using Multiple Description Coding *
}

\author{
Zhipu Jin, Vijay Gupta, Richard M. Murray \\ Division of Engineering and Applied Science, California Institute of Technology, Pasadena
}

\begin{abstract}
For state estimation over a communication network, efficiency and reliability of the network are critical issues. The presence of packet dropping and communication delay can greatly impair our ability to measure and predict states. In this paper, multiple description (MD) codes, a type of network source codes, are used to compensate for this effect on Kalman filtering. We consider two packet dropping models: in one model, packet dropping occurs according to an independent and identically distributed (i.i.d.) Bernoulli random process and in the other model, packet dropping is bursty and occurs according to a Markov chain. We show that MD codes greatly improve the statistical stability and performance of Kalman filter over a large set of packet loss scenarios in both cases. Our conclusions are verified by simulation results.
\end{abstract}

Key words: State estimation, Packet dropping, Kalman filter, Multiple description coding

\section{Introduction}

One of most significant challenges for control theory today is that the control objective is being enlarged from single physical systems to large-scale, complex systems and networks [13]. Tremendous amounts of information need to be sensed, processed and transmitted among different subsystems. Examples include congestion control in routers for the Internet, optimal operation of power grid, air traffic control networks, and many others. Communication networks play an important role in these examples. The theory of networked control systems (NCS) provides an approach to investigate the impact of communication constrains on feedback control systems by replacing the "ideal" feedback links with communication networks. Fig. 1 shows a simplified version of networked control system that omits the communication channel from the controller to the dynamic system. The link from observer to estimator is not modelled as a single, exclusive communication channel, but rather as a possible path through a large, complex communication network shared with many other users.

Efficient and reliable communication requires improvements in both source and channel coding. In addition, dynamical evolution of the system and priori acknowledge of the dynamics can give us extra benefits on top of just using current state-of-the-art communication theory and technology. This merging between control and communication has received considerable interest. Works like $[10,16,17,19,20]$ have focused on answering a fundamental question: how much information at least do we need to send to achieve stability? The main idea in these works is that the uncertainty of the states of the dynamic system changes with respect to time. In order to stabilize the system, the minimum feedback information must be large enough to compensate for the increase in the uncertainty. Other papers, such as $[1,2]$, have studied the sensitivity of the feedback system to quantization noise. They point out that feedback information can be useful even with different levels of resolution.

* This paper was not presented at any IFAC meeting. Corresponding author Z. Jin.

Email addresses: jzp@caltech.edu (Zhipu Jin), vijay@cds.caltech.edu (Vijay Gupta), murray@caltech.edu (Richard M. Murray). 


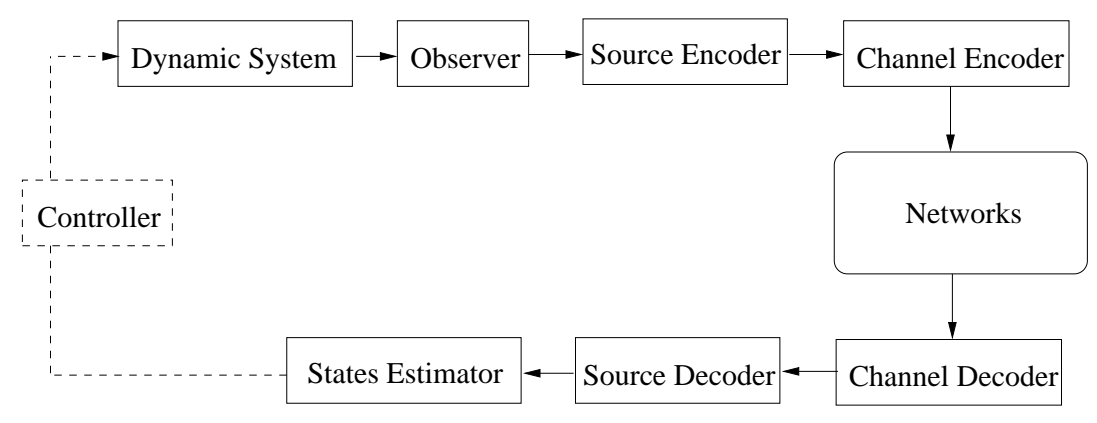

Fig. 1. Diagram of a networked control system.

Most modern communication networks are digital and are implemented using packet-based protocols. Thanks to the incredible developments in communication technology, the ratio of cost to bandwidth of communication links has dropped dramatically. However, the reliability of communication link in networks has become an important issue. Communication networks are not used exclusively for transmitting information between two single points. Packets have to be dropped whenever the network becomes congested. Stochastic packet dropping is very common in large scale networks [21]. Using transmission control protocol (TCP), dropped packets are resent after certain delays. For networked control systems, this protocol does not help if we assume estimator and controller only use available new, "real-time" data packets to update estimation and control laws due to limited memory and computation ability.

In this paper, we ignore the controller in Fig. 1 and focus on the problem of state estimation. Rather than worrying about limited bandwidth, we are concerned about that packets can be dropped by the communication network. We want to understand how the packet dropping affects state estimation and what we can do to compensate for this unreliability?

There are two popular models for packet dropping in large scale networks. The Bernoulli model [21] describes packet losses according to an i.i.d. Bernoulli random process. Another model is the Gilbert-Elliott model [3,6] which describes packet dropping as a Markov chain and is used to handle bursty packet dropping. Sinopoli et al. [15] has used the Bernoulli model to study the statistical convergence properties of the estimation error covariance in a Kalman filter by solving a Modified Algebraic Riccati Equation (MARE). This work shows that packet dropping degrades the performance of Kalman filter. Liu et al. [11] extended the results to the case with partial observation losses in sensor networks. In this paper, we show that multiple description (MD) source codes, a type of network source codes [4], can be used to compensate for the unreliability of communication networks. MD codes have been studied in information theory for over 30 years $[5,8]$ and successfully used in transmission of real-time speech and audio/video over the internet [7,9]. The efficiency of MD codes have been proved in situations where data can be used at various resolution levels. However, this is the first time such coding scheme is applied to networked control systems.

This paper is organized as follows. In Section 2, state estimation problem in networked control systems is described and networking assumptions are given. In Section 3, MD source codes are introduced and theoretical limits are discussed. We formulate the state estimation problem with MD coding in Section 4 and present results for the i.i.d. Bernoulli model. In addition, examples and simulation results are listed. Then the same estimation problem is studied for the Markov chain model in Section 5 and conclusions are summarized in Section 6.

\section{State Estimation Problem and Assumptions}

In this paper, we study the state estimation problem for the following discrete-time linear dynamic system:

$$
\begin{gathered}
x_{k+1}=A x_{k}+w_{k} \\
y_{k}=C x_{k}+v_{k}
\end{gathered}
$$

where $x_{k} \in \mathcal{R}^{n}$ is the state vector, $y_{k} \in \mathcal{R}^{m}$ is the output vector, $w_{k}$ and $v_{k}$ are Gaussian white noise vectors with zero mean and covariance matrices are $Q \geq 0$ and $R>0$ respectively. We assume that $A$ is unstable and use standard discrete-time Kalman filter as the estimator. It is well known that if the pair $\left(A, Q^{\frac{1}{2}}\right)$ is controllable, the 
pair $(A, C)$ is detectable, and no measurements are lost, the estimation error covariance of Kalman filter converges to a unique value from any initial condition.

For the networked control system in Fig. 1, the observation data is put into data packets and is sent through the communication network after going through source and channel encoders. We list some assumptions for the network which simplify our problem:

- We ignore channel coding and assume that the packet will be either received and decoded successfully at the end of the links or totally lost.

- For the estimator, only the new, "real-time" data is used for each update cycle. We only consider the transmission delay that is determined by the network bandwidth and length of the packet. If a packet arrives too late, it is discarded and treated as a dropped packet. So the packet transmission is in a "UPD-like" style which means packet re-sending is not used.

- The network does not provide preferential treatment to any packet. In other words, the network treats each single packet equally without inspecting the content. Thus a multiple resolution code or a layered source code is not a good choice for us since they mark packets with different priorities according to the contents.

- There is no feedback from the decoder to the encoder.

- We assume that number of bits in each data packet is relative large and the network is running at high bit rate scenario.

We assume that packet dropping happens according to one of the two models:

- The i.i.d. Bernoulli model. A Bernoulli random variable $\gamma_{k}$ indicates whether the packet $k$ is received correctly. If it goes through the network successfully, then $\gamma_{k}=1$, otherwise, $\gamma_{k}=0$. For any value of $k, \gamma_{k}$ is independent and identically distributed with probability distribution $P\left(\gamma_{k}=1\right)=\lambda$ and $P\left(\gamma_{k}=0\right)=(1-\lambda)$. This is the simplest and often used model for packet dropping in large scale networks.

- The Gilbert-Elliott channel model. This model considers the network as a discrete-time Markov chain with two possible states - 'good' and 'bad'. In the "good" state, the packet is received correctly, and in the "bad" state, the packet is dropped. The network jumps between these two states according to a Markov chain with transition probability matrix $\mathcal{Q}$ as

$$
\mathcal{Q}=\left[\begin{array}{ll}
q_{00} & q_{01} \\
q_{10} & q_{11}
\end{array}\right]
$$

where 1 is the good state, 0 is the bad state, and $q_{i j}$ is the probability from the previous state $j$ to the next state $i$. The model can be easily extended to more possible states with different packet dropping probabilities. However, for simplicity and without loss of generality, we consider the 2-state model in this paper. Unlike the first model, this one is able to capture the dependence between consecutive losses, i.e., bursty packet dropping.

\section{Multiple Description Source Coding}

For networked control systems, the traditional source code is actually a quantizer $q: \mathcal{R}^{m} \rightarrow \mathcal{Z}$ with a state space partition set $\left\{V_{i}\right\}$ where $i \in(1,2, \cdots, N), \bigcap_{i=1}^{N} V_{i}=\emptyset$, and $\bigcup_{i=1}^{N} V_{i}=\mathcal{R}^{m}$. For each partition $V_{i}$, there exist a codeword $v_{i} \in V_{i}$ and the set of all the codewords is called a codebook. The encoder functions are $f_{e}(x)=i$ if $x \in V_{i}$ and decoder functions are $f_{d}(i)=v_{i}$. We call the integer $i$ the description of the state $x$. The distortion function at the decoder is defined as $d\left(x, v_{i}\right)=\left\|x-v_{i}\right\|^{2}$. Generally, if $N$ is bigger, each partition will be smaller and the average distortion at decoder side is smaller. However, the cost is sending more bits through the network. Rate distortion theory, part of information theory, is used to study any possible partition set and the corresponding average distortion. In this section, we focus on uniform scalar quantizers and assume that the state of the dynamic system is uniformly distributed on a state space whose length is $L$. The optimal distortion rate function for single description source codes is

$$
D(R) \leq \frac{L^{2}}{12} \cdot 2^{-2 R}
$$

where $R=\log _{2}(N)$ is the bits per source sample (bpss) and $N$ is the number of the quantization levels. For other state distributions, we have the similar distortion rate functions that all decay at the speed $2^{-2 R}$.

Multiple description source codes are designed to achieve good rate-distortion performance over lossy links. The unique feature of MD codes is that instead of using one single description to represent one source sample, MD codes 
use two or more descriptions. So at the end of the link, the decoder has much less chance of losing all descriptions. The distortion at the decoder depends on how many descriptions it receives and could be at various quality levels. Also we would like to keep the total bpss as small as possible. Thus the design of a MD code is a problem of minimizing the size of the code over the redundancy between the descriptions. Also MD codes need to be non-hierarchical such that the receiving order of descriptions is not important.

\subsection{Theoretical limits of Multiple Description Codes}

In this subsection, we introduce some theoretical limits for MD codes that fit our discussion on networked control systems. We start with a 2-description MD code. An encoder is fed by a sequence of source sample values $\left\{X_{k}\right\}$. The output of the encoder is $\left\{i_{k}, j_{k}\right\}$. The number of bits for each description are $R_{i}$ and $R_{j}$. There are three cases according to which descriptions are received:

- If for any $k$, the decoder receives none of the descriptions, we call this the "broken link" case. We will discuss this case in Section 4.

- If for any $k$, the decoder receives both $\left\{i_{k}\right\}$ and $\left\{j_{k}\right\}$, we call this the "central decoder" case. In this case, the average distortion is $D_{c}$.

- If for any $k$, the decoder only receives $\left\{i_{k}\right\}$ or $\left\{j_{k}\right\}$, we call this the "side decoder" case. The average distortions are $D_{i}$ and $D_{j}$ respectively.

The main theoretical problem of MD coding is to determine the achievable quintuple $\left(R_{i}, R_{j}, D_{c}, D_{i}, D_{j}\right)$. As discussed in [5], the fundamental tradeoff in MD coding is making descriptions individually good and sufficiently different at the same time. If $R_{i}=R_{j}$ and $D_{i} \approx D_{j}$, then we say the MD code is balanced. The achievable rate-distortion region of a 2-description MD code for a memoryless unit variance Gaussian source with mean-squared error (MSE) distortion is given by [14]:

$$
\left\{\begin{array}{l}
D_{i} \geq 2^{-2 R_{i}} \\
D_{j} \geq 2^{-2 R_{j}} \\
D_{c} \geq 2^{-2\left(R_{i}+R_{j}\right)} \cdot \gamma\left(D_{i}, D_{j}, R_{i}, R_{j}\right)
\end{array}\right.
$$

where

$$
\gamma=\frac{1}{1-\left(\sqrt{\left(1-D_{i}\right)\left(1-D_{j}\right)}-\sqrt{D_{i} D_{j}-2^{-2\left(R_{i}+R_{j}\right)}}\right)^{2}}
$$

for $D_{i}+D_{j}<1+2^{-2\left(R_{i}+R_{j}\right)}$ and $\gamma=1$ otherwise.

For packet-based networked control systems, we use balanced MD codes and assume that $R_{i}=R_{j}=R \gg 1$ and $D_{i}=D_{j} \ll 1$, then we get

$$
\begin{aligned}
\frac{1}{\gamma} & =1-\left(\left(1-D_{i}\right)-\sqrt{D_{i}^{2}-2^{-4 R}}\right)^{2} \\
& \approx 1-\left(\left(1-D_{i}\right)-D_{i}\right)^{2} \approx 4 D_{i}
\end{aligned}
$$

and

$$
D_{c} \cdot D_{i} \geq \frac{1}{4} 2^{-4 R}
$$

The inequality (5) shows the tradeoff between central and side distortions. Compared with inequality (3), it is clear that the penalty in the exponential rate of decay of $D_{i}$ is exactly the increase in the rate of decay of $D_{c}$.

\subsection{Multiple Description Scalar Quantization}

In this section we present the actual design method for MD scalar quantizers (MDSQ). In networked control systems, each source sample is a observation of the dynamic system and MDSQ gives two descriptions for each source sample. This approach was proposed and popularized by Vaishampayan [18] and Fig. 2 shows the diagram of a 2-description MDSQ:

- Step one, select a uniform quantizer with proper step size $\Delta$ and the number of step levels $N$. A source sample $Y$ is quantized by rounding off to the nearest multiple of a step size $\Delta$ and the index output of the uniform quantizer is $n$ which satisfies $0<n \leq N$. 
- Step two, the index $n$ is assigned to a pair of indexes $(i, j)$ by using an index mapping matrix.

- Step three, the $i$ and $j$ are entropy coded and put into data packets respectively.

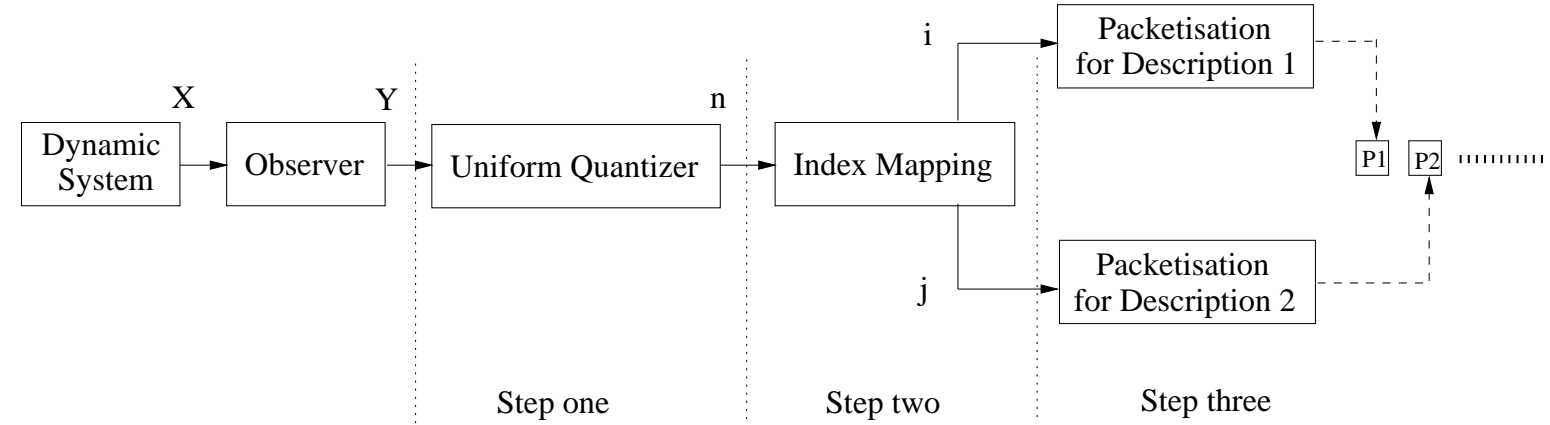

Fig. 2. Diagram of 2-description MD source coding for NCS.

The index mapping problem in step two is the main part of MDSQ. We state this problem as follows: there exists a $\sqrt{M} \times \sqrt{M}$ matrix where $M \geq N$. We need to arrange all these numbers from $\{1, \cdots, N\}$ into the cells of the matrix. Each cell can hold one number at most and each index $n$ gets a pair of matrix index $(i, j)$ according to it's location, i.e., matrix index $i$ is the row number and $j$ is the column number. By this index mapping matrix, step two transfers each single description $n$ into two descriptions $i$ and $j$. Since $N \leq M$, the total number of the possible index assignment methods is $\sum_{n=1}^{M} M ! /(M-n)$ !. The "modified nested" index assignment method is a popular and systematical method and please refer to Appendix A for details. By choosing a proper index mapping matrix, we get

$$
\left\{\begin{array}{l}
D_{c} \approx C_{0} 2^{-2 R(1+\alpha)} \\
D_{i} \approx D_{j} \approx C_{1} 2^{-2 R(1-\alpha)}
\end{array}\right.
$$

where $C_{0}$ and $C_{1}$ are constants that depend on the distribution of the initial state and uniform quantizer in step one. The parameter $\alpha \in[0,1]$ is a pre-defined parameter that indicates the tradeoff between the decay speeds of $D_{c}$ and $D_{i}$. It is clear that the "central decoder" equals a decoder for the uniform quantizer in step one.

The index mapping method can be extended to 3-description or 4-description MD codes. The first part of Table 1 shows some examples of average distortions for different description loss cases when we keep the central distortion constant. It shows that more bits per source sample are needed in order to get the same central decoder distortion. The second part shows that, when we keep bpss constant, the distortion increases when the number of descriptions per sample increases. In the table, "lost $k$ " means $k$ descriptions have been lost, and "N/A" means not available. The table shows that MD coding actually provides various quality levels corresponding to how many descriptions the decoder receives.

Another issue about MD coding is that the computation complexity of decoding increases since the size of the codebook increases at the decoder side as the number of descriptions increases. For example, for a traditional uniform quantizer with $N$ levels, the codebook for the corresponding $L$-description MD code has $\left(2^{L}-1\right) N$ elements. We need to consider this issue when choosing the number of the descriptions per source sample.

\subsection{Quantization Noise of $M D$ codes}

As discussed in [12], the quantization noise of a uniform scalar quantizer with the assumptions of small partition cells, reproduction values at cell's midpoints, and large support region can be approximately modelled as an additive uncorrelated white noise to the quantizer input. For balanced MD codes, the central decoder case actually is a uniform scalar quantizer with the midpoints as the outputs and the average distortion is $D_{c} \approx \frac{\Delta^{2}}{12}$ where $\Delta$ is the length of partition cells. For the side decoder case, index mapping introduces a slight asymmetry between the two side distortions and causes a small increase in distortion. However, for large bpss, this asymmetry asymptotically disappears. According to Section 3, we have

$$
D_{i} \approx D_{j} \approx C_{1} \cdot\left(\frac{C_{2}}{12}\right)^{\frac{1+\alpha}{1-\alpha}} \cdot\left(\Delta^{\frac{1-\alpha}{1+\alpha}}\right)^{2}
$$


Table 1

MSE for different MD codes.

\begin{tabular}{|c|c|c|c|c||c|}
\hline Coding type & No loss & Lost 1 & Lost 2 & Lost 3 & Total bpss \\
\hline \hline single description & $8.33 \times 10^{-6}$ & N/A & N/A & N/A & 10 \\
\hline 2-description & $8.33 \times 10^{-6}$ & 1.56 & N/A & N/A & 12 \\
\hline 3-description & $8.33 \times 10^{-6}$ & $4.41 \times 10^{-3}$ & 1.53 & N/A & 15 \\
\hline 4-description & $8.33 \times 10^{-6}$ & $7.46 \times 10^{-3}$ & $1.34 \times 10^{-2}$ & 2.61 & 20 \\
\hline
\end{tabular}

\begin{tabular}{|c|c|c|c|c||c|}
\hline Coding type & No loss & Lost 1 & Lost 2 & Lost 3 & Total bpss \\
\hline \hline single description & $4.97 \times 10^{-7}$ & N/A & N/A & N/A & 12 \\
\hline 2-description & $8.33 \times 10^{-6}$ & 1.56 & N/A & N/A & 12 \\
\hline 3-description & $9.87 \times 10^{-5}$ & $1.97 \times 10^{-2}$ & 2.15 & N/A & 12 \\
\hline 4-description & $9.32 \times 10^{-4}$ & $8.04 \times 10^{-2}$ & 0.113 & 2.18 & 12 \\
\hline
\end{tabular}

For a balanced 2-description MD code, $\alpha$ is a constant and $D_{i}$ will be asymptotically negligible relative to $\left(\Delta^{\frac{1+\alpha}{1-\alpha}}\right)^{2}$. So as long as the bit rate $R_{i}\left(=R_{j}\right)$ is big enough, the additive noise model is still a good approximation to represent the quantization noise in the side decoder case. From now on, we model the MD quantization noise as Gaussian white noise with zero mean and covariance $D_{c}$ for central decoder case and $D_{i}$ for side decoder case.

\section{Kalman Filtering Utilizing MD with i.i.d. Packet Dropping}

\subsection{Problem Formulation}

We consider the discrete-time linear dynamical system described by equations (1) and assume that packet dropping is independent and is described by an i.i.d. Bernoulli random process. We use a 2-description balanced MD code. Each element $y_{k}$ of the measurement output $Y_{k}$ is encoded by two descriptions $\left\{i_{k}, j_{k}\right\}$. We organize these descriptions into 2 description vectors as $\left\{I_{k}, J_{k}\right\}$ and put them into two packets. Variables $\gamma_{I, k}$ and $\gamma_{J, k}$ are used to indicate whether the description vectors $I_{k}$ and $J_{k}$ are received correctly. If $I_{k}$ is received correctly, then $\gamma_{I, k}=1$, otherwise, $\gamma_{I, k}=0$, and similarly for $\gamma_{J, k}$. We assume that $\gamma_{I, k}$ and $\gamma_{J, k}$ are i.i.d. Bernoulli random variables with probability distribution $P\left(\gamma_{I, k}=1\right)=P\left(\gamma_{J, k}=1\right)=\lambda$.

Since $\gamma_{I, k}$ and $\gamma_{J, k}$ are independent, we have three measurement rebuilding scenarios. First, we may receive both the descriptions correctly. In this case, the measurement noise is the white noise $v_{t}$ plus the central distortion noise. We use $R_{0}=R+D_{c}$ to indicate the covariance where $D_{c}$ is the central distortion covariance. Second, we may receive only one description correctly and the measurement noise is $R_{1}=R+D_{i}$ where $D_{i}$ is the side distortion covariance. Third, we may receive none of the descriptions correctly. In this case, we assume the measurement is corrupted by an infinitely large noise. This is corresponding to the "broken link" case in Section 3 . The noise is changed into a random variable $\hat{v}_{t}$ after the decoder and the covariance $C_{k}$ is:

$$
C_{k}=\left\{\begin{array}{l}
R_{0}: \text { probability is } \lambda^{2} \\
R_{1}: \text { probability is } 2(1-\lambda) \lambda \\
\sigma^{2} I: \text { probability is }(1-\lambda)^{2}
\end{array}\right.
$$

where $\sigma \rightarrow \infty$.

The Kalman filter recursion thus becomes stochastic and the error covariance evolves as

$$
\begin{aligned}
P_{k+1} & =A P_{t} A^{\prime}+Q \\
& \left.\left.-\gamma_{(} I, k\right) \gamma_{(} J, k\right) A P_{k} C^{\prime}\left[C P_{k} C^{\prime}+R_{0}\right]^{-1} C P_{k} A^{\prime} \\
& \left.\left.-\left(1-\gamma_{(} I, k\right)\right) \gamma_{(} J, k\right) A P_{k} C^{\prime}\left[C P_{k} C^{\prime}+R_{1}\right]^{-1} C P_{k} A^{\prime} \\
& \left.\left.-\gamma_{(} I, k\right)\left(1-\gamma_{(} J, k\right)\right) A P_{k} C^{\prime}\left[C P_{k} C^{\prime}+R_{1}\right]^{-1} C P_{k} A^{\prime}
\end{aligned}
$$


Thus, the sequence of the error covariance matrix $P_{k=0}^{\infty}$ is a random process for any given initial value. Using the same approach as in [15], we define the Modified Algebraic Riccati Equation (MARE) for Kalman filter using balanced 2-description MD codes as:

$$
\begin{aligned}
g_{\lambda}(X)= & A X A^{\prime}+Q \\
& -\lambda^{2} A X C^{\prime}\left(C X C^{\prime}+R_{0}\right)^{-1} C X A^{\prime} \\
& -2(1-\lambda) \lambda A X C^{\prime}\left(C X C^{\prime}+R_{1}\right)^{-1} C X A^{\prime}
\end{aligned}
$$

and the expected value of error covariance matrix $E\left[P_{k}\right]$ evolves according to this MARE.

\subsection{Statistical Convergence Properties}

This subsection lists theorems which describe the convergence properties of the MARE in equation (8). These theorems are based on the lemmas in Appendix B. The first theorem listed below states the uniqueness of the MARE solution.

Theorem 1 Consider the operator

$$
\phi\left(K_{0}, K_{1}, X\right)=(1-\lambda)^{2}\left(A X A^{\prime}+Q\right)+\lambda^{2}\left(F_{0} X F_{0}^{\prime}+V_{0}\right)+2(1-\lambda) \lambda\left(F_{1} X F_{1}^{\prime}+V_{1}\right)
$$

where $F_{0}=A+K_{0} C, F_{1}=A+K_{1} C, V_{0}=Q+K_{0} R_{0} K_{0}^{\prime}$, and $V_{1}=Q+K_{1} R_{1} K_{1}^{\prime}$. Suppose there exist $K_{0}, K_{1}$, and $P>0$ such that $P>\phi\left(K_{0}, K_{1}, P\right)$. Then we have

(a) for any initial condition $P_{0} \geq 0, M A R E$ (8) converges, i.e. the iteration $P_{k+1}=g_{\lambda}\left(P_{k}\right)$ converges, and the limit is independent of the initial value:

$$
\lim _{k \rightarrow \infty} P_{k}=\lim _{k \rightarrow \infty} g_{\lambda}^{k}\left(P_{0}\right)=\bar{P}
$$

(b) $\bar{P}$ is the unique positive semi-definite solution of MARE function $\bar{P}=g_{\lambda}(\bar{P})$.

Proof (a) First, we show that the MARE converges with initial value $Q_{0}=0$. Let $Q_{k}=g_{\lambda}\left(Q_{k-1}\right)=g_{\lambda}^{k} g_{\lambda}\left(Q_{0}\right)$, then $Q_{1} \geq Q_{0}=0$ and

$$
Q_{1}=g_{\lambda}\left(Q_{0}\right) \leq g_{\lambda}\left(Q_{1}\right)=Q_{2}
$$

By induction, we know that the sequence $\left\{Q_{k}\right\}$ is nondecreasing. Also by lemma $14,\left\{Q_{k}\right\}$ is bounded and there exists an $M_{Q_{0}}$ such that $Q_{k} \leq M_{Q_{0}}$ for any $k$. Therefore, the sequence converges and

$$
\lim _{k \rightarrow \infty} Q_{k}=\bar{P}
$$

where $\bar{P}$ is a fixed point of the iteration $\bar{P}=g_{\lambda}(\bar{P})$.

Next we show that the iteration $\bar{R}_{k}=g_{\lambda}^{k}\left(\bar{R}_{0}\right)$ initialized at $R_{0} \geq \bar{P}$ also converges to $\bar{P}$. Since $R_{1}=g_{\lambda}\left(R_{0}\right) \geq$ $g_{\lambda}(\bar{P})=\bar{P}$, then $R_{k} \geq \bar{P}$ for any $k$. Also

$$
\begin{aligned}
0 \geq R_{k+1}-\bar{P} & =g_{\lambda}\left(R_{k}\right)-g_{\lambda}(\bar{P}) \\
& =\phi\left(K_{R_{k} 0}, K_{R_{k} 1}, R_{k}\right)-\phi\left(K_{\bar{P} 0}, K_{\bar{P} 1}, \bar{P}\right) \\
& \leq \phi\left(K_{\bar{P} 0}, K_{\bar{P} 1}, R_{k}\right)-\phi\left(K_{\bar{P} 0}, K_{\bar{P} 1}, \bar{P}\right) \\
& =\mathcal{L}\left(R_{k}-\bar{P}\right) .
\end{aligned}
$$

According to lemma 13, for any $Y \geq 0$,

$$
\lim _{k \rightarrow \infty} \mathcal{L}^{k}=0
$$

So we get $0 \geq \lim _{k \rightarrow \infty}\left(R_{k}-\bar{P}\right)=0$, i.e. the sequence $R_{k}$ converges to $\bar{P}$. 
Then we show that, for any initial condition $P_{0} \geq 0$, the iteration $P_{k}=g_{\lambda}^{k}\left(P_{0}\right)$ converges to $\bar{P}$. Let $R_{0}=P_{0}+\bar{P} \geq \bar{P}$, then $0 \leq Q_{0} \leq P_{0} \leq R_{0}$, by induction, we have $0 \leq Q_{k} \leq P_{k} \leq R_{k}$. Since $\left\{Q_{k}\right\}$ and $\left\{R_{k}\right\}$ converges to $\bar{P}$, so

$$
\lim _{k \rightarrow \infty} P_{k}=\bar{P} \text {. }
$$

(b) Suppose there exists another positive semi-definite $\hat{P}$ such that $\hat{P}=g_{\lambda}(\hat{P})$. Then let the iteration be initialized at $\hat{P}$ and generate a new sequence $\{\hat{P} \cdots \hat{P}\}$ which should converges to $\bar{P}$. Thus $\hat{P}=\bar{P}$.

The following theorem states the conditions for MARE convergence.

Theorem 2 If $\left(A, Q^{\frac{1}{2}}\right)$ is controllable, $(A, C)$ is detectable, and $A$ is unstable, then there exists a $\lambda_{c} \in[0,1)$ such that

(a) $\lim _{k \rightarrow \infty} E\left[P_{k}\right]=+\infty$ for $0 \leq \lambda \leq \lambda_{c}$ and some initial condition $P_{0} \geq 0$,

(b) $E\left[P_{k}\right] \leq M_{P_{0}} \forall k$ for $\lambda_{c}<\lambda \leq 1$ and any initial condition $P_{0} \geq 0$,

where $M_{P_{0}}>0$ depends on the initial condition $P_{0}$.

Proof Please refer to Appendix B

This theorem claims that there exists a critical value $\lambda_{c}$ of the packet receiving probability. If $\lambda$ is smaller than $\lambda_{c}$, MARE (8) does not converge and the expected value of error covariance matrix will diverge.

Theorem 3 Let

$$
\begin{aligned}
\underline{\lambda} & =\arg \inf _{\lambda}\left[\exists \hat{S} \mid \hat{S}=(1-\lambda)^{2} A \hat{S} A^{\prime}+Q\right]=1-\frac{1}{\alpha} \\
\bar{\lambda} & =\arg \inf _{\lambda}\left[\exists \hat{X} \mid \hat{X}>g_{\lambda}(\hat{X})\right] \\
& =\arg \inf _{\lambda}\left[\exists\left(\hat{K}_{0}, \hat{K}_{1}, \hat{X}\right) \mid \hat{X}>\phi\left(\hat{K}_{0}, \hat{K}_{1}, \hat{X}\right)\right]
\end{aligned}
$$

where $\alpha=\max \left|\sigma_{i}\right|$ and $\sigma_{i}$ are the eigenvalues of $A$. Then

$$
\underline{\lambda} \leq \lambda_{c} \leq \bar{\lambda} .
$$

Proof Please refer to Appendix B

This theorem states the upper and lower bounds for $\lambda_{c}$. The lower bound is in a closed form. According to the next theorem and corollary, we can reformulate the computation of $\bar{\lambda}$ as an LMI feasible problem.

Theorem 4 Assume that $\left(A, Q^{\frac{1}{2}}\right)$ is controllable and $(A, C)$ is detectable, then the following statements are equivalent:

(a) $\exists \bar{X}$ such that $\bar{X}>g_{\lambda}(\bar{X})$;

(b) $\exists\left(\bar{K}_{0}, \bar{K}_{1}, \bar{X}\right)>0$ such that $\bar{X}>\phi\left(\bar{K}_{0}, \bar{K}_{1}, \bar{X}\right)$;

(c) $\exists \bar{Z}_{0}, \bar{Z}_{1}$ and $0<\bar{Y} \leq I$ such that $\Psi_{\lambda}\left(\bar{Y}, \bar{Z}_{0}, \bar{Z}_{1}\right)>0$ where

$$
\begin{gathered}
\Psi_{\lambda}\left(Y, Z_{0}, Z_{1}\right)=\left[\begin{array}{cccc}
Y & \Delta\left(Y, Z_{1}\right) & \Omega\left(Y, Z_{0}\right) & \Pi(Y) \\
\Delta\left(Y, Z_{1}\right)^{\prime} & Y & 0 & 0 \\
\Omega\left(Y, Z_{0}\right)^{\prime} & 0 & Y & 0 \\
\Pi(Y)^{\prime} & 0 & 0 & Y
\end{array}\right], \\
\Delta\left(Y, Z_{1}\right)=\sqrt{2(1-\lambda) \lambda}\left(Y A+Z_{1} C\right), \Omega\left(Y, Z_{0}\right)=\lambda\left(Y A+Z_{0} C\right), \text { and } \Pi(Y)=(1-\lambda) Y A .
\end{gathered}
$$


Corollary 5 The upper bound $\bar{\lambda}$ is given by the solution of the following optimization problem,

$$
\bar{\lambda}=\arg \min _{\lambda}\left(\Psi_{\lambda}\left(Y, Z_{0}, Z_{1}\right)>0\right)
$$

where $0<Y \leq I$.

Proof Please refer to Appendix B for the proof.

When $C$ is invertible, we choose $K_{0}=K_{1}=-A C^{-1}$ to make $F_{0}=F_{1}=0$ and the LMI in theorem 4 is equivalent to

$$
X-(1-\lambda)^{2} A X A^{\prime}>0 .
$$

Since $X \geq 0$ exists if and only if $(1-\lambda) A$ is stable, i.e. all the magnitudes of eigenvalues of $(1-\lambda) A$ are smaller than 1, we obtain $\bar{\lambda}=\underline{\lambda}=\frac{1}{1-\alpha}$. According to [15], the lower bound of using single description codes is $1-\frac{1}{\alpha^{2}}$ which is bigger than using MD codes. Also if either $C$ is invertible or the quantization noise $D_{c}$ and $D_{i}$ are smaller than $R$ (which is always true for the high bpss case), it is easy to show that the upper bound of using single description codes is also bigger than using MD codes. So using MD codes pushes $\lambda_{c}$ to a smaller value and guarantee the convergence over a larger packet dropping scenario.

The following theorem gives the upper and lower bounds on the expected value of error covariance matrix when MARE converges. The lower bound $\bar{S}$ can be computed by standard Lyapunov Equation Solvers and the upper bound $\bar{V}$ can be either computed via iterating $V_{t+1}=g_{\lambda}\left(V_{t}\right)$ from any initial condition or transferred to a semi-definite programming (SDP) problem.

Theorem 6 Assume $\left(A, Q^{\frac{1}{2}}\right)$ is controllable, $(A, C)$ is detectable, and $\bar{\lambda}<\lambda$, then for any initial condition $E\left[P_{0}\right] \geq$ 0 ,

$$
0 \leq \bar{S} \leq \lim _{k \rightarrow \infty} E\left[P_{k}\right] \leq \bar{V}
$$

where $\bar{S}$ and $\bar{V}$ are solutions of the equations $\bar{S}=(1-\lambda)^{2} A \bar{S} A^{\prime}+Q$ and $\bar{V}=g_{\lambda}(\bar{V})$ respectively.

Proof Let $S_{k+1}=\mathcal{M}\left(S_{k}\right)=(1-\lambda)^{2} A S_{k} A^{\prime}+Q$ and $V_{k+1}=g_{\lambda}\left(V_{k}\right)$ with initial conditions $S_{0}=0$ and $V_{0}=E\left[P_{0}\right] \geq$ 0 . By induction and theorem 3 , we obtain

$$
S_{k} \leq E\left[P_{k}\right] \leq V_{k}
$$

for any $t$. According to theorem $1, \lim _{k \rightarrow \infty} V_{k}=\bar{V}$ where $\bar{V}=g_{\lambda}(\bar{V})$. Also since $\left(A, Q^{\frac{1}{2}}\right)$ is controllable and all the magnitudes of the eigenvalues of $(1-\lambda) A$ are smaller than 1 , the sequence of the Lyapunov iteration converges to the strictly positive definite solution of the Lyapunov function, i.e. $\lim _{k \rightarrow \infty} S_{k}=\bar{S}>0$. Therefore we can conclude that

$$
0<\bar{S}=\lim _{k \rightarrow \infty} S_{k} \leq \lim _{k \rightarrow \infty} E\left[P_{k}\right] \leq \lim _{k \rightarrow \infty} V_{k}=\bar{V} .
$$

\subsection{Simulation Results}

In this subsection simulation results are given to verify the advantages of MD codes. We choose the discrete time LTI system with $A=-1.25$ and $C=1$. The noises $w_{t}$ and $v_{t}$ have zero means and covariances $R=2.5$ and $Q=1$ respectively. A balanced 2-description MD code is designed such that the central distortion $D_{0} \approx 8.33 \times 10^{-6}$ and the side distortion $D_{1} \approx 1.56$. The bpss of the MD code is 12 and the bpss of a single description code with the same distortion is 10 bits.

Fig. 3 shows the expected error covariance with the theoretical upper and lower bounds. The simulations are run 1000 times and we use the average values as the expected values. The asymptote $\lambda_{c}$ has been pushed from 0.36 for the single description code to 0.2 for the MD code. Convergence properties of error covariance at high packet loss rate region are also improved dramatically. Note that when $\lambda$ is close to the asymptote, some of the simulated error covariances values are below the lower bound. The reason is that we only take limited time steps for the simulation (2000 steps each) and some expected error covariances take longer time to converge. 


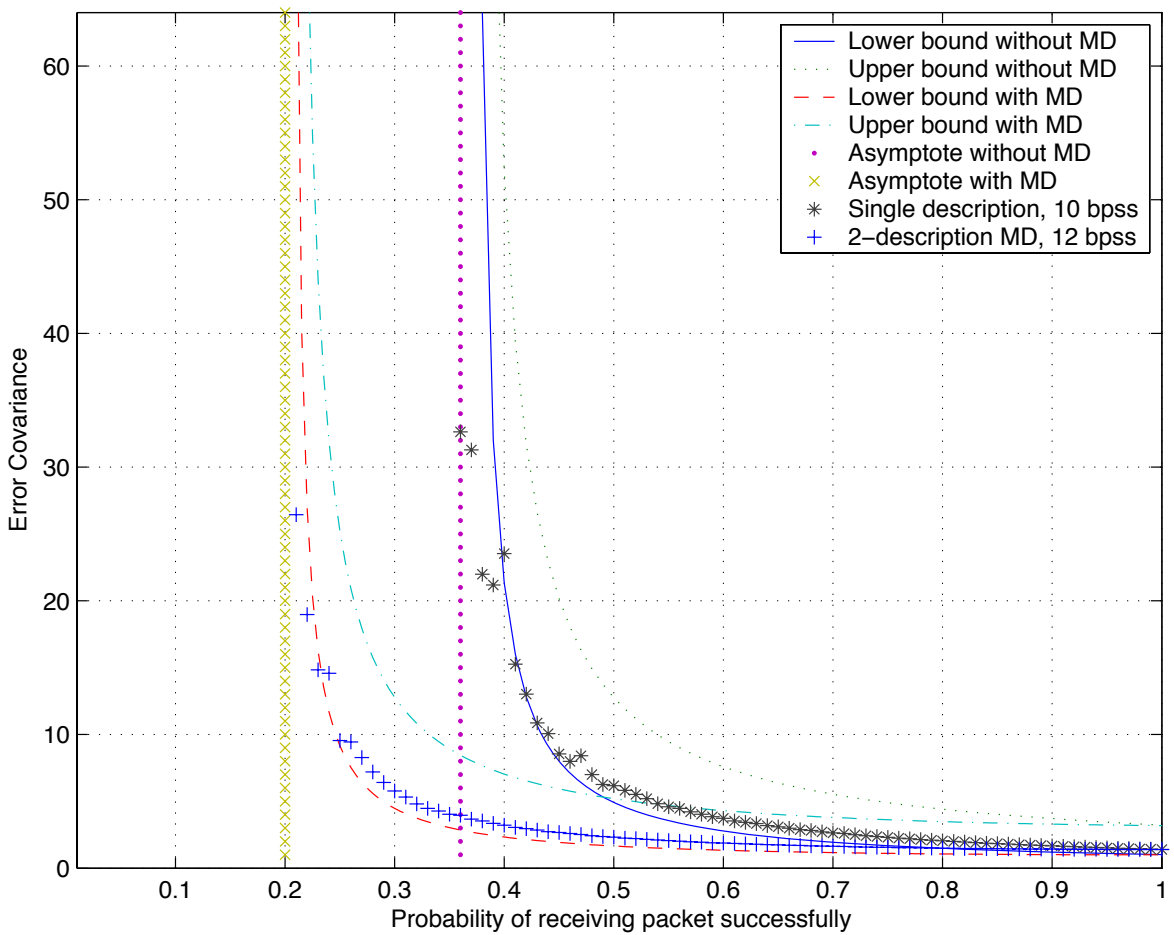

Fig. 3. Simulation results of expected error covariances with theoretical upper and lower bounds.

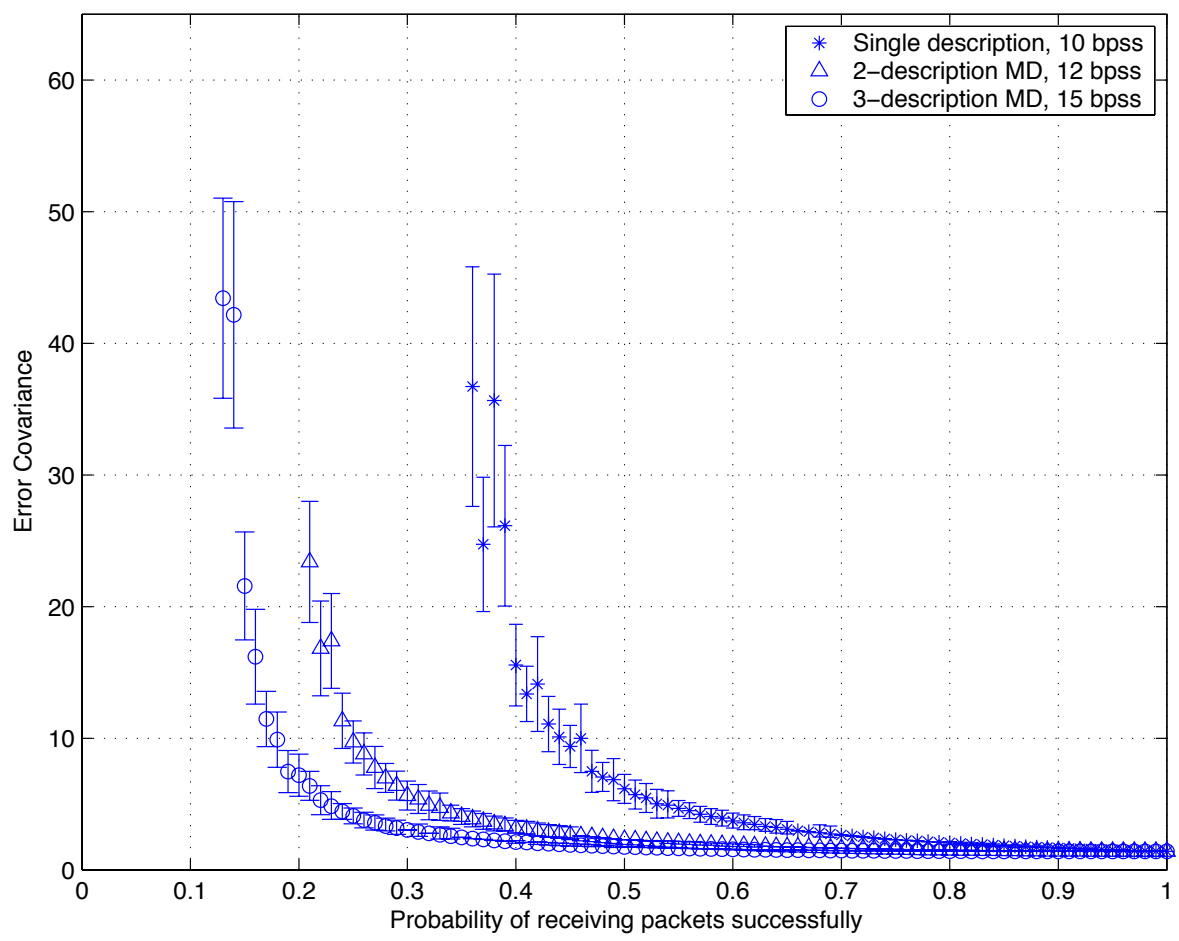

Fig. 4. Mean values of error covariance with same central distortions. 


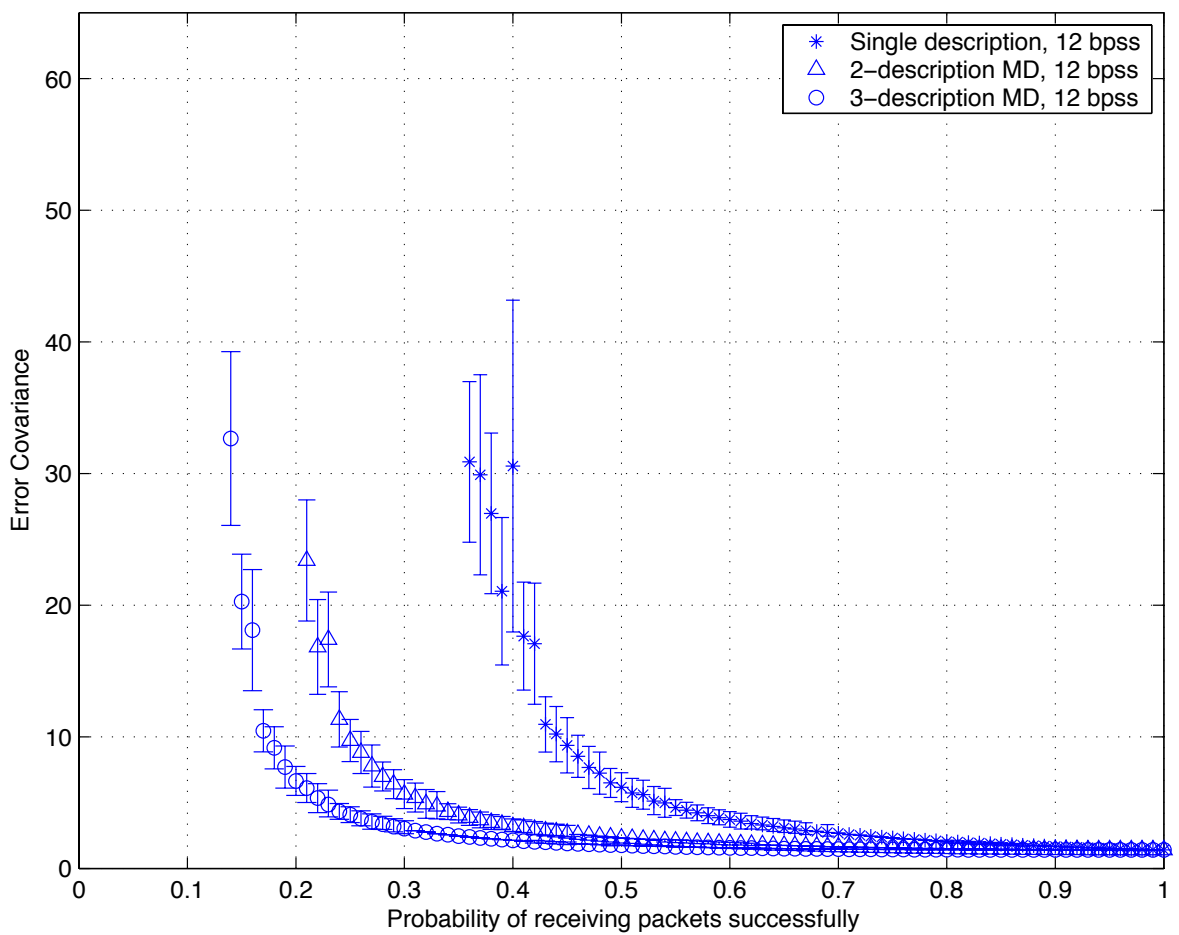

Fig. 5. Mean values of error covariance with same bpss.

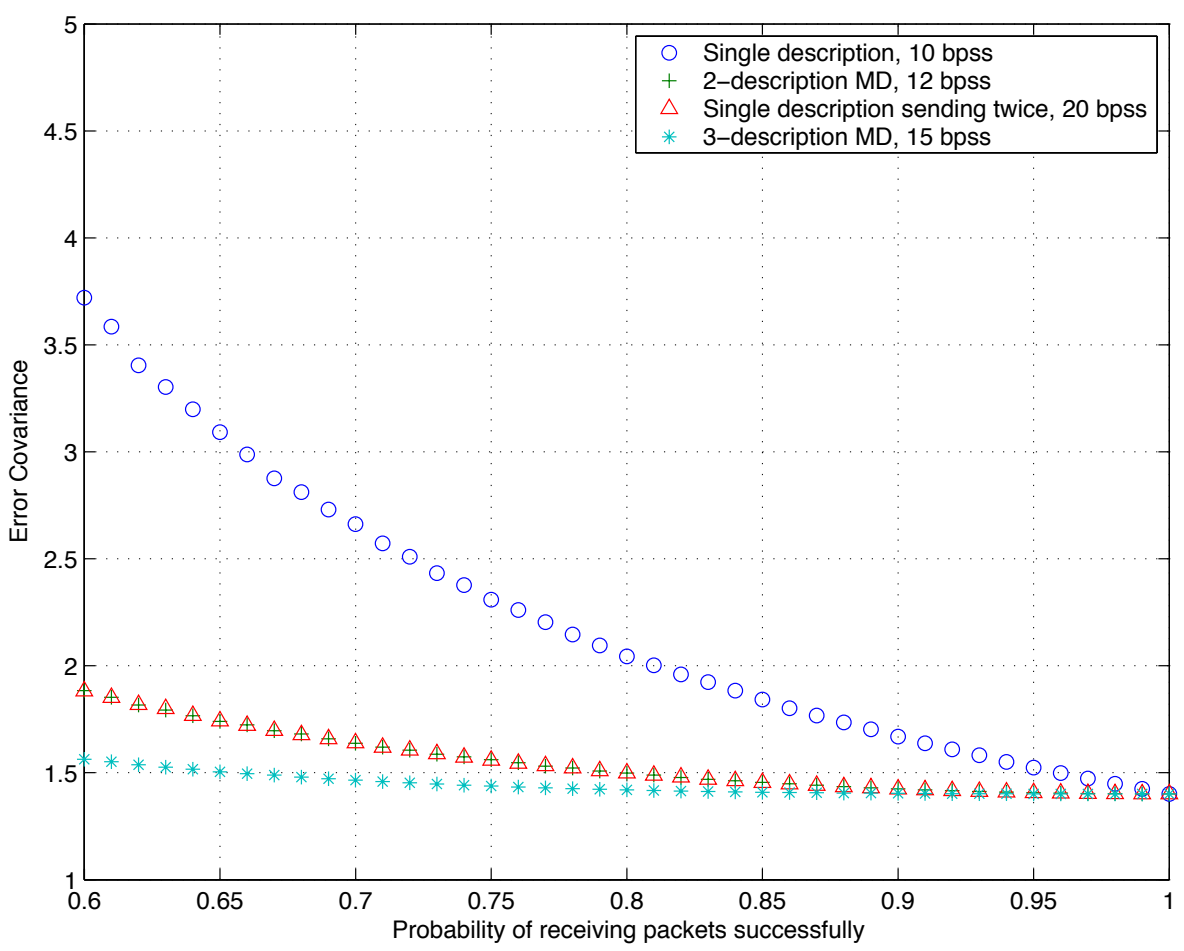

Fig. 6. Mean values of error covariance with low dropping rate. 
Fig. 4 shows some other simulation results. For each packet dropping rate, the centers of the error bars are the mean values and $95 \%$ of the simulation results are located inside the error bars. It shows that, if we use a balanced 3-description MD code, the critical value $\lambda_{c}$ is even smaller. So the benefits of using MD codes are clear and the cost we need to pay is more bits for each source sample. It can be shown that when $C$ is invertible, using a $L$-description MD code we can get $\lambda_{c}=1-\alpha^{-2 / L}$. Unfortunately, finding an optimal $L$-description MD code for arbitrary $L$ is still an open problem in information theory. When we keep bpss constant, as shown in Fig 5 , we get bigger quantization noise as the number of descriptions increases. Compared with the previous figure, there are no obvious differences due to the accuracy loss in this example.

In some cases, packet dropping rate of a practical communication network is fairly small. Fig 6 shows the expected error covariance when packet dropping rate is low and MD codes give much better performance than the single description code. Note that the 2-description MD code achieves as good performance as sending single description code twice but saves up to $40 \%$ bandwidth.

\section{$5 \quad$ Kalman Filtering Utilizing MD with Bursty Packet Dropping}

\subsection{Convergence Conditions and Boundaries}

As discussed in Section 2, a way to model the bursty packet dropping is using a 2-state Markov chain with transition probability matrix $\mathcal{Q}$ given by equation (2). For the case of a balanced $2-\mathrm{MD}$ code, we are thus interested in a 4 -state Markov chain where the states correspond to both packets lost, only the 1st description packet lost, only the 2nd description packet lost and no packet lost. The transition probability matrix of this chain is given as

$$
\mathcal{Q}_{M D}=\left[\begin{array}{lccc}
q_{00}^{2} & q_{00}^{2} & q_{01} q_{00} & q_{01} q_{00} \\
q_{01} q_{10} & q_{01} q_{10} & q_{11} q_{01} & q_{11} q_{01} \\
q_{10} q_{00} & q_{10} q_{00} & q_{01} q_{10} & q_{01} q_{10} \\
q_{10} q_{11} & q_{10} q_{11} & q_{11}^{2} & q_{11}^{2}
\end{array}\right]
$$

Note that the state in which both description packets are lost is equivalent to no observation coming through, while the other states correspond to the system being observed. If the Markov chain is stationary, the state probabilities tend to a stationary distribution as $k \rightarrow \infty$. However, we normally cannot directly study the problem over the steady-state distribution since this distribution might not be achievable. This problem mathematically equals the random sensor selection problem in sensor networks. Consider the system

$$
x_{k+1}=A x_{k}+w_{k}
$$

being observed through $n$ sensors with the $i$-th sensor of the form

$$
y_{k}^{i}=C^{i} x_{k}+v_{k}^{i}
$$

Suppose only one sensor can be active at any time instant and the choice of the sensor is done according to a Markov chain. We denote the Ricatti update in error covariance by $f_{i}($.$) when the i$-th sensor is used and denote

$$
f_{i}^{k}(.)=\underbrace{f_{i}\left(f_{i}(\cdots(.) \cdots)\right)}_{k \text { times }} .
$$

The expected error covariance at time step $k$ is denoted by $E\left[P_{k}\right]$. Probability of the network in Markov state $j$ at time $k$ is denoted by $\pi_{k}^{j}$ and $q_{i j}$ is the probability of the network state is $i$ at time $k+1$ given the network state is $j$ at time $k$.

Lemma 7 For any Ricatti update operator $f_{i}(P)$, we have

(a) $f_{i}(P) \geq Q$; 
(b) If $X<Y$, then $f_{i}(X) \leq f_{i}(Y)$;

(c) $f_{i}(P)$ is concave w.r.t. $P$.

When a single description code is applied, according to equation (12), packet dropping can be treated as the observation jumps between 2 sensors which have the same $C^{i}$ matrices and different Gaussian noises with covariance $R_{0}$ and $\sigma^{2} I$ respectively where $\sigma \rightarrow \infty$. The Kalman filter error covariance updates are

$$
\left\{\begin{array}{l}
f_{0}(P)=A P A^{\prime}+Q \\
f_{1}(P)=A P A^{\prime}+Q-A P C^{\prime}\left(C P C^{\prime}+R_{0}\right)^{-1} C P A^{\prime}
\end{array}\right.
$$

Similar to the i.i.d. Bernoulli model, we discuss the conditions and the upper/lower bounds for expected values of estimation error covariances converging.

Theorem 8 When using a single-description code and with the Markov probability transition matrix given by equation (2), the lower bound for $E\left[P_{k}\right]$ is $Y_{k}$ where

$$
\begin{aligned}
Y_{k}= & q_{00}^{k} \pi_{0}^{0} f_{0}^{k}\left(P_{0}\right) \\
& +\sum_{i=1}^{k-1} q_{00}^{i}\left(\pi_{k+1-i}^{0}-q_{00} \cdot \pi_{k-i}^{0}\right) f_{0}^{i}(Q)+\pi_{k}^{1} f_{1}(Q) .
\end{aligned}
$$

The upper bound is $X_{k}$ where

$$
X_{k}=\sum_{j=0}^{1} \sum_{i=0}^{1} f_{j}\left(X_{k-1}^{i}\right) q_{j i} \pi_{k-1}^{i}
$$

and $X_{k-1}^{i}=E\left[X_{k-1} \mid\right.$ state is $i$ at time $\left.(k-2)\right]$.

proof Suppose $k$ starts from 1 , and for any $k$, we define event $E_{i}$ as last packet was received at time $k-i$ where $i \in[0, \cdots, k]$. So the probability of $E_{i}$ is

$$
p_{i}= \begin{cases}q_{00}^{k} \pi_{0}^{0} & i=k \\ q_{00}^{i-1} q_{01} \pi_{k-i}^{1} & 0<i<k \\ \pi_{k}^{1} & i=0\end{cases}
$$

and the error covariance $P_{k}$ if $E_{i}$ happens is

$$
P_{k} \mid E_{i}= \begin{cases}f_{0}^{k}\left(P_{0}\right) & i=k \\ f_{0}^{i}\left(f_{1}\left(P_{k-i}\right)\right) & 0<i<k \\ f_{1}\left(P_{k}\right) & i=0\end{cases}
$$

So

$$
\begin{aligned}
E\left[P_{k}\right] & =\sum_{i=0}^{k} p_{i} \cdot P_{k} \mid E_{i} \\
& =q_{00}^{k} \pi_{0}^{0} f_{0}^{k}\left(P_{0}\right)+\sum_{i=1}^{k-1} q_{00}^{i} q_{01} \pi_{k-i}^{1} f_{0}^{i}\left(f_{1}\left(P_{k-i}\right)\right)+\pi_{k}^{1} f_{1}\left(P_{k}\right) .
\end{aligned}
$$

According to Lemma $7, f_{1}\left(P_{k-1}\right) \geq Q$, so

$$
\begin{aligned}
E\left[P_{k}\right] & \geq q_{00}^{k} \pi_{0}^{0} f_{0}^{k}\left(P_{0}\right)+\sum_{i=1}^{k-1} q_{00}^{i} q_{01} \pi_{k-i}^{1} f_{0}^{i}(Q)+\pi_{k}^{1} f_{1}(Q) \\
& =q_{00}^{k} \pi_{0}^{0} f_{0}^{k}\left(P_{0}\right)+\sum_{i=1}^{k-1} q_{00}^{i}\left(\pi_{k+1-i}^{0}-q_{00} \cdot \pi_{k-i}^{0}\right) f_{0}^{i}(Q)+\pi_{k}^{1} f_{1}(Q)
\end{aligned}
$$


For upper bound, let us denote $S_{k}$ is the network state at time $k$. For single description code, $S_{k} \in[0,1]$. Then $E\left[P_{k}\right]=\sum_{j=0}^{1} \pi_{k}^{j} \cdot E\left[P_{k} \mid S_{k}=j\right]$. Also

$$
\begin{aligned}
\pi_{k}^{j} \cdot E\left[P_{k} \mid S_{k}=j\right] & =\pi_{k}^{j} \sum_{i=0}^{1} E\left[P_{k} \mid S_{k}=j, S_{k-1}=i\right] \cdot p\left(S_{k-1}=i \mid S_{k}=j\right) \\
& =\sum_{i=0}^{1} E\left[f_{j}\left(P_{k-1}\right) \mid S_{k-1}=i\right] q_{j i} \pi_{k-1}^{i} \\
& \leq \sum_{i=0}^{1} f_{j}\left(\left[P_{k-1} \mid S_{k-1}=i\right]\right) q_{j i} \pi_{k-1}^{i}
\end{aligned}
$$

since $f_{j}(\cdot)$ is concave.

Proposition 9 A sufficient condition for divergence of expected error covariance is:

$$
q_{00} \cdot \alpha^{2}>1
$$

where $\alpha=\max \left|\sigma_{i}\right|$ and $\sigma_{i}$ are the eigenvalues of $A$.

Using a balanced 2-MD code, the corresponding sensor selection problem has 4 sensors which have same $C$ matrices and noise covariances are $R_{0}, R_{1}, R_{1}$, and $\sigma^{2} I$ respectively. The Ricatti updates are

$$
\left\{\begin{array}{l}
f_{0}(P)=A P A^{\prime}+Q \\
f_{1}(P)=f_{2}(P)=A P A^{\prime}+Q-A P C^{\prime}\left(C P C^{\prime}+R_{1}\right)^{-1} C P A^{\prime} . \\
f_{3}(P)=A P A^{\prime}+Q-A P C^{\prime}\left(C P C^{\prime}+R_{0}\right)^{-1} C P A^{\prime} .
\end{array}\right.
$$

Using the same approach, we get

Proposition 10 When using 2-description code and with the underlying Markov probability transition matrix given by (2), the lower bound for $E\left[P_{k}\right]$ is $Y_{k}$ where

$$
\begin{aligned}
Y_{k}= & q_{00}^{2 k} \pi_{0}^{0} f_{0}^{k}\left(P_{0}\right) \\
& +\sum_{i=1}^{k-1} q_{00}^{2 i}\left(\pi_{k+1-i}^{0}-q_{00}^{2} \cdot \pi_{k-i}^{0}\right) f_{0}^{i}(Q)+\sum_{j=1}^{3} \pi_{k}^{j} f_{j}(Q) .
\end{aligned}
$$

The upper bound is $X_{k}$ where

$$
X_{k}=\sum_{j=0}^{3} \sum_{i=0}^{3} f_{j}\left(X_{k-1}^{i}\right) q_{j i} \pi_{k-1}^{i}
$$

and $X_{k-1}^{i}=E\left[X_{k-1} \mid\right.$ state is $i$ at time $\left.(k-2)\right]$.

A sufficient condition for divergence of expected error covariance is:

$$
q_{00} \cdot \alpha>1
$$

The equations for lower and upper bounds are pretty messy but can be calculated iteratively. Also, these bounds are dependent on value of $q_{11}$ and initial distribution of packet dropping $\pi_{0}^{i}$.

\subsection{Simulation Results}

We use the same LTI system as in Section 4. In figure 7 we plot the theoretical upper and lower bounds for the error variance as a function of $q_{10}$ under the conditions as $q_{11}=0.95$ and uniform distribution of $\pi_{0}^{i}$. The lowering of the bounds is indicative of the performance getting better with MD codes. The simulation results with parameters $q_{11}=0.05$ and $q_{11}=0.95$ with different coding schemes are shown in figure 8 . 


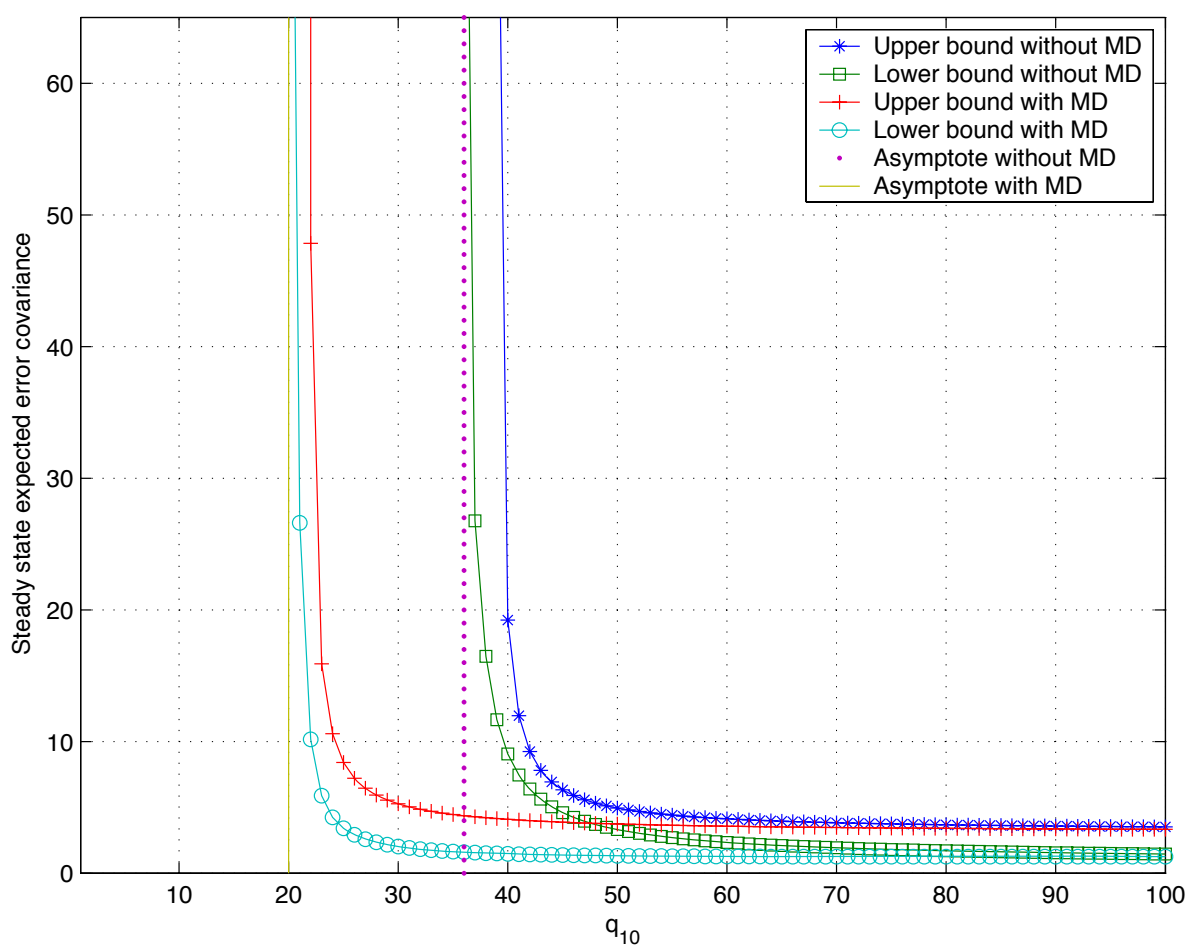

Fig. 7. Theoretical upper and lower bounds for burst packet dropping case with $q_{11}=95 \%$.

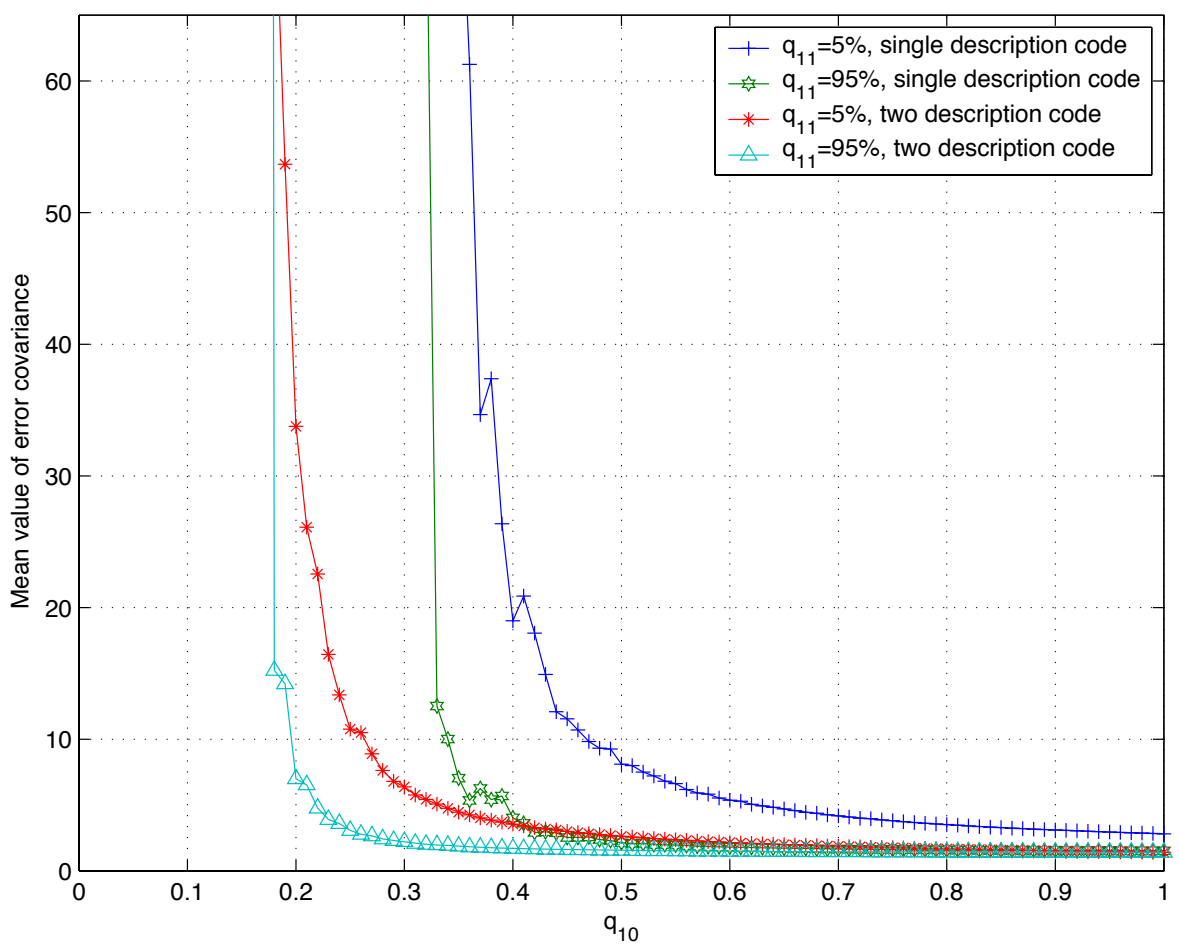

Fig. 8. Simulation results for burst packet dropping case. 
In the Markov chain model, $q_{01}=1-q_{11}$ is a measure of the frequency of the bursty dropping while $q_{00}=1-q_{10}$ indicates how big the burst dropping is. According to the simulation results, expected error covariance diverges more quickly with higher $q_{01}$. This makes sense since if the error bursts happen often, we expect the estimation error covariance to diverge. It is seen from the figures that the system diverges around $q_{10}=0.36$ with the single description code case, and diverges around $q_{10}=0.2$ with the 2-MD code. (Limited simulation time steps make the results smaller than lower bounds near asymptotes.) Thus the stability margin is enlarged if we use MD codes. Also, for same $q_{10}$, using MD codes greatly decreases the expected error covariance.

\section{Conclusion}

In this paper, we present a new coding method for state estimation problem counter-acting the effect of packet dropping: using multiple description source coding. The accuracy of the decoder only depends on how many descriptions are successfully received. We considered two typical network packet dropping models: the i.i.d. Bernoulli model and the Gilbert-Elliott model. Using MD codes, the convergence region of the estimation error covariance is much larger than using traditional single description code and the steady expected values are also much smaller. Moreover, MD code is an optimal code which saves considerable bandwidth than sending duplicated packets.

The main goal of this paper is trying to understand the impact of communication constrains from another angle: in high bit rate scenario and with large, complex communication networks, what can we do to compensate for packet loss as well as to satisfy the real-time demands? In this papere, we have to compromise the accuracy of the source code to improve the convergency properties of MARE. This is a good demonstration to indicate the close relationship between communication theory and control theory when we face the challenges in "an information rich world" [13].

There are several promising research directions for the future. From the communication theory side, a more general theory and design method for MD coding for arbitrary number of description is needed. Also, since using MD codes greatly increases the computation complexity of the decoder, a more efficient search algorithm for decoding is needed. From the feedback control theory side, the stability and robustness of close-loop networked control systems with MD coding need to be studied.

\section{Acknowledgements}

The authors would like to thank Prof. M. Effros for the inspiring ideas of MD coding. They also want to thank B. Hassibi, A. Tiwari, and D. G. MacMynowski for valuable discussions and comments. This work is supported in part by AFOSR grant FA9550-04-1-0169 and NSF-ITR grant CCR-0326554.

\section{References}

[1] R. W. Brockett and D. Liberzon. Quantized feedback stabilization of linear systems. IEEE Trans. Automat. Contr., 45(7):1279-1289, July 2000.

[2] N. Elia and S. K. Mitter. Stabilization of linear systems with limited information. IEEE Trans. Automat. Contr., 46(9):1384-1400, Sep. 2001.

[3] E. O. Elliott. Estimates of error rates for codes on burst-noise channels. Bell Syst. Tech. J., 42:1977-1997, Sept. 1963.

[4] M. Fleming, Q. Zhao, and M. Effros. Network vector quantization. IEEE Trans. Inform. Theory, 50(8):1584-1604, 2004.

[5] A. A. El Gamal and T. M. Cover. Achievable rates for multiple descriptions. IEEE Trans. Inform. Theory, 28(6):851-857, 1982.

[6] E. N. Gilbert. Capacity of a burst-noise channel. Bell Syst. Tech. J., 39:1253-1265, Sept. 1960.

[7] V. K. Goyal, J. Kovaceric, R. Arean, and M. Vetterli. Multiple description transform coding of images. In Proceedings of IEEE International Conference on Image Processing, volume 1, pages 674-678, Chicago, IL, Oct. 1998.

[8] V. K. Goyal and J. Kovacevic. Generalized multiple description coding with correlating transforms. IEEE Trans. Inform. Theory, 47(6):2199-2224, 2001.

[9] W. S. Lee, M. R. Pickering, M. R. Frater, and J. F. Arnold. A robust codec for transmission of very low bit-rate video over channels with bursty errors. IEEE Trans. Circuits Syst. Video Technol., 10:1403-1412, Dec. 2000.

[10] D. Liberzon. On stabilization of linear systems with limited information. IEEE Trans. Automat. Contr., 48(2):304-307, Feb. 2003.

[11] X. Liu and A. J. Goldsmith. Kalman filtering with partial observation losses. Paradise Island, Bahamas, Dec. 2004. IEEE Conference on Decision and Control.

[12] D. Marco and D. L. Neuhoff. The validity of the additive noise model for uniform scalar quantizers. IEEE Trans. Inform. Theory, Submitted. 
[13] R. M. Murray, editor. Control in an Information Rich World (Panel Report). 2002.

[14] L. Ozarow. On a source-coding problem with two channels and three receivers. Bell Syst. Tech. J., 59(10):1909-1921, 1980.

[15] B. Sinopoli, L. Schenato, M. Franceschetti, K. Poolla, M. I. Jordan, and S. S. Sastry. Kalman filtering with intermittent observations. IEEE Trans. Automat. Contr., 49(9):1453-1464, Sept. 2004.

[16] S. Tatikonda and S. K. Mitter. Control over noisy channels. IEEE Trans. Automat. Contr., 49(7):1196-1201, July 2004.

[17] S. Tatikonda and S. K. Mitter. Control under communication constraints. IEEE Trans. Automat. Contr., 49(7):1056-1068, July 2004.

[18] V. A. Vaishampayan. Design of multiple description scalar quantizers. IEEE Trans. Inform. Theory, 39(3):821-834, 1993.

[19] G. C. Walsh, H. Ye, and L. G. Bushnell. Stability analysis of networked control systems. IEEE Trans. Contr. Syst. Technol., 10(3):438-446, May 2002.

[20] W. S. Wong and R. W. Brockett. Systems with finite communication bandwidth constraints - part I: State estimation problems. IEEE Trans. Automat. Contr., 42(9):1294-1299, September 1997.

[21] M. Yajnik, S. Moon, J. Kurose, and D. Towsley. Measurement and modelling of the temporal dependence in packet loss. In INFOCOM '99. Eighteenth Annual Joint Conference of the IEEE Computer and Communications Societies. Proceedings. IEEE, volume 1, pages 345-352, New York, NY, USA, March 1999.

\section{A The "Modified Nested" Index Assignment Method for MDSQ}

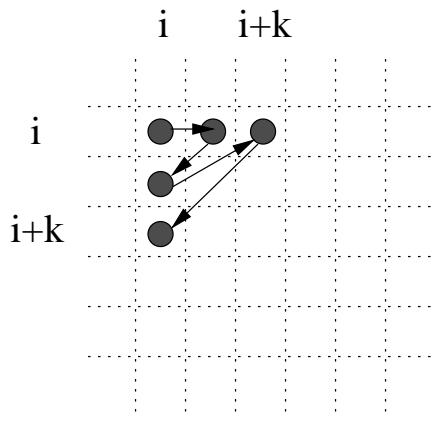

(a)

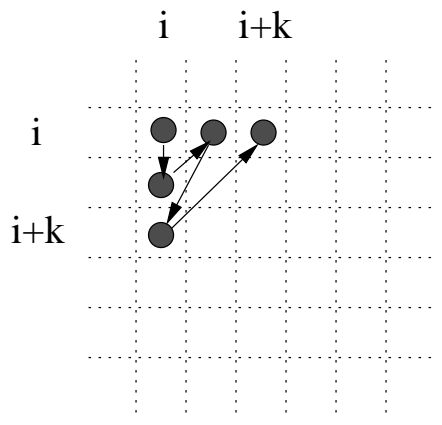

(b)

Fig. A.1. Two actions of MD index mapping: part(a) is the action $\bar{E}_{i}$ and part (b) is the action $\bar{S}_{i}$

The "modified nested" index assignment method in [18] is a tractable and systematical method. From 1 to $N$, the index $n$ is placed on the main diagonal and the $2 k$ diagonals that lie closest to the main diagonal, from left-upper corner to right-lower corner. Note that $0<k<\sqrt{M}$, let $k_{i}=\min (k, \sqrt{M}-i)$. Define the action $\bar{E}_{i}$ as putting index $n$ into the sequence of matrix cells $(i, i),(i, i+1),(i+1, i),(i, i+2),(i+2, i), \cdots,\left(i, i+k_{i}\right),\left(i+k_{i}, i\right)$ and the action $\bar{S}_{i}$ as putting index $n$ into the sequence of matrix cells $(i, i),(i+1, i),(i, i+1),(i+2, i),(i, i+2), \cdots,\left(i+k_{i}, i\right)$, $\left(i, i+k_{i}\right)$. Fig. A.1 shows these two different actions.

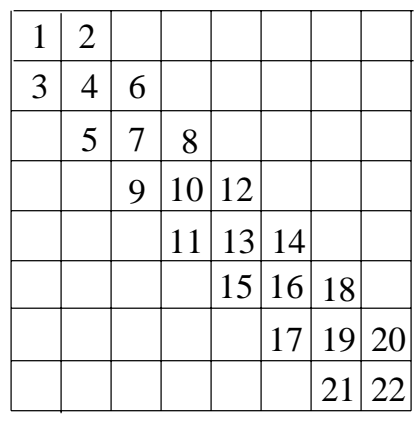

(a)

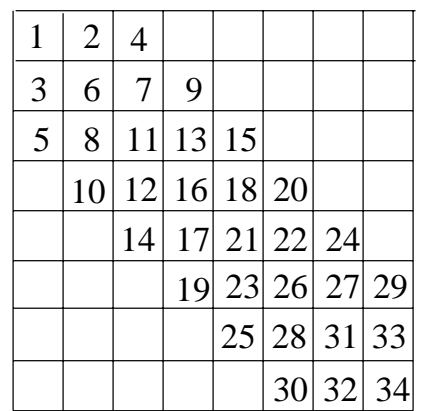

(b)

Fig. A.2. Index mapping for: $\operatorname{part}(\mathrm{a}) M=64, k=1$ and part (b) $M=64, k=2$

From $n=1$ and matrix cell $(i, i)=(1,1)$, we repeat action $\bar{E}_{i} k$ times, then repeat action $\bar{S}_{i} k$ times, then switch 
to action action $\bar{E}_{i}$ for $k$ times again and continue this process until we reach $(i, i)=(\sqrt{M}, \sqrt{M})$. Then we have:

$$
\left\{\begin{array}{l}
N=(2 k+1) \sqrt{M}-k(k+1) \\
R=R_{i}=R_{j}=\log _{2}(\sqrt{M}) \\
R_{c}=\log _{2}(N)
\end{array}\right.
$$

where $R_{c}$ is the bit number per symbol value of the uniform quantizer in step one. For given $k$ and $R$, we use $M N(R, k)$ to denote the MD code. Let $\sqrt{M}=k^{1 / \alpha}$ where $0<\alpha<1$. When $M$ and $N$ is large, i.e., at high bit rate, we can get these relationships about the central and side distortion:

$$
\left\{\begin{array}{l}
D_{c} \approx C_{0} 2^{-2 R(1+\alpha)} \\
D_{i} \approx C_{1} 2^{-2 R(1-\alpha)}
\end{array}\right.
$$

where $C_{0}$ and $C_{1}$ are constants. There exists two extreme cases:

- When $\alpha$ approaches $1, D_{c}$ decreases at the maximum rate of $2^{-4 R}$ while both $D_{i}$ and $D_{j}$ exhibit zero decay rates. In this case, the matrix is almost full and $M \approx N$;

- When $\alpha$ approaches $0, D_{c}, D_{i}$ and $D_{j}$ decay at the same rate of $2^{-2 R}$. In this case, the matrix is only filled in the main diagonal.

Intermediate rates can be achieved by selecting other values of $\alpha$.

\section{B Lemmas and Some Proofs of MARE Convergence with i.i.d. Packet Dropping}

Those theorems in Section 4 are based on these lemmas which can be easily proved by using similar approach in [15]. We list the lemmas here and omit the proofs.

Lemma 11 Let the operator

$$
\phi\left(K_{0}, K_{1}, X\right)=(1-\lambda)^{2}\left(A X A^{\prime}+Q\right)+\lambda^{2}\left(F_{0} X F_{0}^{\prime}+V_{0}\right)+2(1-\lambda) \lambda\left(F_{1} X F_{1}^{\prime}+V_{1}\right)
$$

where $F_{0}=A+K_{0} C, F_{1}=A+K_{1} C, V_{0}=Q+K_{0} R_{0} K_{0}^{\prime}$, and $V_{1}=Q+K_{1} R_{1} K_{1}^{\prime}$. Assume $X \in S=\left\{S \in \mathcal{R}^{n \times n} \mid S \geq\right.$ $0\}, R_{0}>0, R_{1}>0, Q>0$, and $\left(A, Q^{\frac{1}{2}}\right)$ is controllable. Then the following facts are true:

(a) With $K_{x 0}=-A X C^{\prime}\left(C X C^{\prime}+R_{0}\right)^{-1}$ and $K_{x 1}=-A X C^{\prime}\left(C X C^{\prime}+R_{1}\right)^{-1}, g_{\lambda}(X)=\phi\left(K_{x 0}, K_{x 1}, X\right)$;

(b) $g_{\lambda}(X)=\min _{\left(K_{0}, K_{1}\right)} \phi\left(K_{0}, K_{1}, X\right) \leq \phi\left(K_{0}, K_{1}, X\right) \forall\left(K_{0}, K_{1}\right)$;

(c) If $X \leq Y$, then $g_{\lambda}(X) \leq g_{\lambda}(Y)$;

(d) If $\lambda_{1} \leq \lambda_{2}$, then $g_{\lambda_{1}}(X) \geq g_{\lambda_{2}}(X)$;

(e) If $\alpha \in[0,1]$, then $g_{\lambda}(\alpha X+(1-\alpha) Y) \geq \alpha g_{\lambda}(X)+(1-\alpha) g_{\lambda}(Y)$;

(f) $g_{\lambda}(X) \geq(1-\lambda)^{2} A X A^{\prime}+Q$;

(g) If $\bar{X} \geq g_{\lambda}(\bar{X})$, then $\bar{X} \geq 0$;

(h) If $X$ is a random variable, then $(1-\lambda)^{2} A E[X] A^{\prime}+Q \leq E\left[g_{\lambda}(X)\right] \leq g_{\lambda}(E[X])$.

Lemma 12 Let $X_{k+1}=h\left(X_{k}\right)$ and $Y_{k+1}=h\left(Y_{k}\right)$. If $h(X)$ is a monotonically increasing function, then:

$$
\begin{gathered}
X_{1} \geq X_{0} \Rightarrow X_{k+1} \geq X_{k}, \forall k \geq 0 \\
X_{1} \leq X_{0} \Rightarrow X_{k+1} \leq X_{k}, \forall k \geq 0 \\
X_{0} \leq Y_{0} \Rightarrow X_{k} \leq Y_{k}, \forall k \geq 0
\end{gathered}
$$

Lemma 13 Define the linear operator $\mathcal{L}(Y)=(1-\lambda)^{2} A Y A^{\prime}+\lambda^{2} F_{0} Y F_{0}^{\prime}+2(1-\lambda) \lambda F_{1} Y F_{1}^{\prime}$ and suppose there exists $\bar{Y}>0$ such that $\bar{Y}>\mathcal{L}(\bar{Y})$.

(a) For all $W \geq 0, \lim _{k \rightarrow \infty} \mathcal{L}^{k}(W)=0$; 
(b) Let $V \geq 0$ and consider the linear system $Y_{k+1}=\mathcal{L}\left(Y_{k}\right)+V$ initial at $Y_{0}$, then the sequence $\left\{Y_{k}\right\}$ is bounded.

Lemma 14 Suppose there exists $\bar{K}_{0}, \bar{K}_{1}$, and $\bar{P}>0$ such that

$$
\bar{P}>\phi\left(\bar{K}_{0}, \bar{K}_{1}, \bar{P}\right),
$$

then for any initial value $P_{0}$, the sequence $P_{k}=g_{\lambda}^{k}\left(P_{0}\right)$ is bounded, i.e. there exists $M_{P_{0}} \geq 0$ dependent of $P_{0}$ such that

$$
P_{k} \leq M_{P_{0}}, \forall k .
$$

Proof of Theorem 2 Obviously there are two special cases:

- When $\lambda=1$, then the MARE reduces to the standard Algebraic Riccati Equation and it converges to a unique positive semi-definite solution.

- When $\lambda=0$, then all the packets are lost. Since $A$ is unstable, the covariance matrix diverges for some initial values.

Next, we need to show that there exists a single point of transition between the two cases. Suppose for $0<\lambda_{1} \leq 1$, $E_{\lambda_{1}}\left[P_{k}\right]$ is bounded for any initial values. Then for any $\lambda_{2}>\lambda_{1}$, we have

$$
E_{\lambda_{1}}\left[P_{k}\right]=E\left[g_{\lambda_{1}}\left(P_{k}\right)\right] \geq E\left[g_{\lambda_{2}}\left(P_{k}\right)\right]=E_{\lambda_{2}}\left[P_{k}\right] .
$$

So $E_{\lambda_{2}}\left[P_{k}\right]$ is also bounded. Now we can choose

$$
\lambda_{c}=\left\{\inf \lambda^{*}: \lambda>\lambda^{*} \Rightarrow E_{\lambda}\left[P_{k}\right] \text { is bounded for any initial value } P_{0} \geq 0\right\}
$$

and finish the proof.

Proof of Theorem 3 For the lower bound of $\lambda_{c}$, we define the Lyapunov operator $\mathcal{M}(X)=\bar{A} X \bar{A}^{\prime}+Q$ where $\bar{A}=(1-\lambda) A$. If $\left(A, Q^{\frac{1}{2}}\right)$ is controllable, $\left(\bar{A}, Q^{\frac{1}{2}}\right)$ is also controllable. Then the $\hat{S}=\mathcal{M}(\hat{S})$ has a unique strictly positive definite solution $\hat{S}$ if and only if $\max _{i}\left|\sigma_{i}(\bar{A})\right|<1$, so we get $\underline{\lambda}=1-\frac{1}{\alpha}$. Consider the iteration $S_{t+1}=\mathcal{M}\left(S_{t}\right)$ for any $\lambda>\underline{\lambda}$, it converges. While for $\lambda \leq \underline{\lambda}$, it is unstable and $S_{k}$ tends to infinity for any initial values.

For the mean value of the error covariance matrix $E\left[P_{k}\right]$ initialized at $E\left[P_{0}\right] \geq 0$, consider $0=S_{0} \leq E\left[P_{0}\right]$, it's true that

$$
\begin{aligned}
S_{k} \leq E\left[P_{k}\right] \Rightarrow S_{k+1} & =\mathcal{M}\left(S_{k}\right) \\
& \leq(1-\lambda)^{2} A E\left[P_{k}\right] A^{\prime}+Q \\
& \leq E\left[g_{\lambda}\left(P_{k}\right)\right]=E\left[P_{k+1}\right] .
\end{aligned}
$$

By induction, it is obvious that when $\lambda<\underline{\lambda}, \lim _{k \rightarrow \infty} E\left[P_{k}\right] \geq \lim _{k \rightarrow \infty} S_{k}=\infty$. This implies that for any initial condition $E\left[P_{k}\right]$ is unbounded for $\lambda<\underline{\lambda}$, therefore $\underline{\lambda} \leq \lambda_{c}$.

For the upper bound of $\lambda_{c}$, consider the sequence $V_{k+1}=g_{\lambda}\left(V_{k}\right)$ and $V_{0}=E\left[P_{0}\right] \geq 0$, we have

$$
\begin{aligned}
E\left[P_{k}\right] \leq V_{k} \Rightarrow E\left[P_{k+1}\right] & =E\left[g_{\lambda}\left(P_{k}\right)\right] \\
& \leq g_{\lambda}\left(E\left[P_{k}\right]\right) \\
& \leq g_{\lambda}\left(V_{k}\right)=V_{k+1}
\end{aligned}
$$

A simple induction shows that for any $k, V_{k} \geq E\left[P_{k}\right]$. So for $\lambda>\bar{\lambda}$, according to lemma 11 part (g), there exists $\bar{X}>0$. Therefore all conditions of lemma 14 are satisfied and we have

$$
E\left[P_{k}\right] \leq V_{k} \leq M_{V_{0}}
$$


for any $k$. This shows that $\lambda_{c} \leq \bar{\lambda}$.

Proof of Theorem 4 Using lemma 11, it is easy to show that if $\bar{X}>g_{\lambda}(\bar{X})$ exists, then $\bar{X}>0$ and $\bar{X}>g_{\lambda}(\bar{X})=$ $\phi\left(K_{\bar{X} 0}, K_{\bar{X} 1}, \bar{X}\right)$. Also it is clear that $\bar{X}>\phi\left(K_{0}, K_{1}, \bar{X}\right) \geq g_{\lambda}(\bar{X})$, so (a) is equivalent to (b). The only trick we need for the remaining proof is to use Schur complement decomposition to obtain the function $\Psi_{\lambda}$.

$$
\begin{aligned}
\phi\left(K_{0}, K_{1}, X\right)= & (1-\lambda)^{2}\left(A X A^{\prime}+Q\right)+\lambda^{2}\left(F_{0} X F_{0}^{\prime}+V_{0}\right) \\
& +2(1-\lambda) \lambda\left(F_{1} X F_{1}^{\prime}+V_{1}\right) \\
= & (1-\lambda)^{2} A X A^{\prime}+Q+\lambda^{2} F_{0} X F_{0}^{\prime}+2(1-\lambda) \lambda F_{1} X F_{1}^{\prime} \\
& +\lambda^{2} K_{0} R_{0} K_{0}^{\prime}+2(1-\lambda) \lambda K_{1} R_{1} K_{1}^{\prime}
\end{aligned}
$$

The part (b) is equivalent to

$$
\left[\begin{array}{cc}
X-(1-\lambda)^{2} A X A^{\prime}+\lambda^{2} F_{0} X F_{0}^{\prime} & \sqrt{2(1-\lambda) \lambda} F_{1} \\
\sqrt{2(1-\lambda) \lambda} F_{1}^{\prime} & X^{-1}
\end{array}\right]>0
$$

Using Schur complement decomposition two more times to obtain

$$
\left[\begin{array}{cccc}
X & \sqrt{2(1-\lambda) \lambda} F_{1} & \lambda F_{0} & (1-\lambda) A \\
\sqrt{2(1-\lambda) \lambda} F_{1}^{\prime} & X^{-1} & 0 & 0 \\
\lambda F_{0}^{\prime} & 0 & X^{-1} & 0 \\
(1-\lambda) A^{\prime} & 0 & 0 & X^{-1}
\end{array}\right]>0
$$

Let $Y=X^{-1}, Z_{1}=X^{-1} K_{1}$, and $Z_{1}=X^{-1} K_{1}$, we get

$$
\left[\begin{array}{cccc}
Y & \sqrt{2(1-\lambda) \lambda}\left(Y A+Z_{1} C\right) & \lambda\left(Y A+Z_{0} C\right) & (1-\lambda) Y A \\
\sqrt{2(1-\lambda) \lambda(}\left(A^{\prime} Y+C^{\prime} Z_{1}^{\prime}\right) & Y & 0 & 0 \\
\lambda\left(A^{\prime} Y+C^{\prime} Z_{0}^{\prime}\right) & 0 & Y & 0 \\
(1-\lambda) A^{\prime} Y & 0 & 0 & Y
\end{array}\right]>0
$$

and this is what we define as $\Psi_{\lambda}\left(Y, Z_{0}, Z_{1}\right)$. Since $\Psi_{\lambda}\left(\alpha Y, Z_{0}, Z_{1}\right)=\alpha \Psi_{\lambda}\left(Y, Z_{0}, Z_{1}\right)$, so $Y$ can be restricted to $0<Y \leq I$. 